\title{
Proceedings of the Association of British Neurologists from the joint meeting with the British Society for Clinical Neurophysiology, Charing Cross Hospital, 18-20 September 1996
}

CRYPTIC COELIAC DISEASE PRESENTING WITH NEUROMUSCULAR DYSFUNCTION

A Gibson, $M$ Hadjivassiliou, AK Chattopadhyay, GAB Davies-Jones, Royal Hallamshire Hospital, Sheffield, UK

Eight patients are described who presented with neuromuscular abnormalities of obscure aetiology despite thorough investigation. None had evidence of CNS involvement at presentation. All patients had antigliadin antibodies in their serum. Distal duodenal biopsies in all patients fulfilled the histopathological criteria for coeliac disease (CD). Patients ranged in age from 18-67 (median 62) years and there were five men and three women. Neuromuscular disease had been present for 1-18 (median four) years before the diagnosis of $C D$.

All patients had nerve conduction studies and EMG and two patients had muscle biopsy. Three patients had sensorimotor neuropathy, one had motor neuropathy, one had myositis and sensorimotor neuropathy, and one had myositis and motor neuropathy. The neuropathic abnormalities in these six patients were distally predominant and implied axonal pathology. One other patient had mononeuritis multiplex, and one patient had neuromyotonia.

Cryptic CD may initially present with neuromuscular dysfunction. CD merits early consideration in patients with neuromuscular abnormalities of obscure aetiology, especially if introduction of a gluten free diet results in symptomatic improvement.

AN INHIBITOR OF MATRIX

METALLOPROTEINASE ACTIVITY AND TNF $\alpha$ RELEASE IS AN EFFECTIVE TREATMENT IN EXPERIMENTAL AUTOIMMUNE NEURITIS KJ Smith, EJ Redford, $M$ Davies, N Gregson, P Hughes, AJH Gearing, K Miller, RAC Hughes, Guy's Hospital, London, and Neures Ltd, Abingdon, UK

The factors involved in the genesis of the inflammatory autoimmune demyelinating disease Guillain-Barré syndrome (GBS) remain poorly understood, although there is evidence that the pro-inflammatory cytokine tumour necrosis factor- $\alpha$ (TNF $\alpha$ ) may be involved. Because TNF $\alpha$ release is dependent upon a metalloproteinase (MMP)-like enzyme, and since the extracellular degrading MMPs are also implicated in the pathogenesis of autoimmune disease, it was examined whether an inhibitor (BB1101) of this class of enzymes is effective in the therapy of an animal model of GBS. Experimental allergic neuritis (EAN) was induced in 116 Lewis rats using bovine peripheral nerve myelin. When BB1101 was administered by intraperitoneal ( $1 \mathrm{mg} / \mathrm{kg}$, twice daily) or subcutaneous $(10 \mathrm{mg} / \mathrm{kg}$, once daily) routes from the time of immunisation, it prevented the development of the neurological deficits and histological changes observed in vehicle treated controls. Subcutaneous treatment from either day 7 , or from the onset of the disease, again reduced disease severity. BB1101 did not inhibit the development of an immune response, as measured by the production of antimyelin antibodies. It is concluded that MMP inhibitors may have potential as therapeutic agents in peripheral demyelinating diseases such as GBS.

MEASURING SINGLE COGNITIVE EVENTS WITH WHOLE BRAIN FUNCTIONAL MAGNETIC RESONANCE IMAGING

M Humberstone, S Clare, J Hykin, PG Morris, GV Sawle, University of Nottingham, Nottingham, UK

A technique has been developed for studying single cognitive events with functional MRI. The technique uses echoplanar imaging at a field strength of 3 Tesla and generates one functional image of the whole brain every three seconds. A signal averaging technique was used to create time sweeps of functional signal change after a single cognitive event. A $t$ test is used to identify areas and times of significant activation without making any a priori assumptions about the spatial or temporal pattern of response; thus allowing both to be characterised. This technique was used to analyse functional activity during simple motor tasks in six normal subjects. Widely distributed patterns of functional activity appeared transiently 3-6 seconds after a single externally cued press of a button with the right thumb. Activation was seen in primary and in premotor cortex in all subjects. The time course of activation can be examined in real time using video. This technique can be used to analyse and compare the separate tasks which constitute a cognitive paradigm. Comparison between decisions to move and decisions not to move in a "go", "no-go" paradigm discloses hierarchical organisation of the mesial premotor cortex and identifies "decision making" and "motor execution" areas.

\section{MONITORING PAPILLOEDEMA WITH} CONFOCAL LASER TOMOGRAPHY IN IDIOPATHIC INTRACRANIAL HYPERTENSION DA Mulholland, J Craig, JM Gibson, SJA Rankin, Royal Victoria Hospital, Belfast, UK

This study measured the efficacy of confocal laser tomography at assessing optic disc swelling in patients with idiopathic intracranial hypertension. Eight patients with papilloedema were studied. At every examination three images were acquired, each comprising 32 transverse optica sections of the optic disc at a depth of 1.5 to 4.0. A topographic map is created. A contour is drawn around the swollen disc to enable comparison of subsequent images to baseline.

Eight patients were examined regularly over a six to 12 month period. Five patients with symptomatic improvement on medical treatment showed significant reduction in papilloedema (neuroretinal rim volume) and nerve fibre layer thickness. Four of these had resolution of visual field defects; one was unreliable at perimetry. Three patients with chronic disc swelling and mild field loss showed no improvement or worsening of papilloedema and nerve fibre layer thickness; disc changes were not eviden clinically. Automated Humphrey $30^{\circ}$ perimetry was used.

This technique is a sensitive measure of optic disc changes, quantifies swelling, and is a useful adjunct to perimetry in monitoring idiopathic intracranial hypertension, particularly if perimetry is unreliable. Several methods of displaying images are demonstrated.

MANAGING IDIOPATHIC INTRACRANIAL. HYPERTENSION: COORDINATED FOLLOW UP IS ESSENTIAL

J Craig, D Mulholland, JM Gibson, Royal Victoria Hospital, Belfast, UK

The optimum management of patients with idiopathic intracranial hypertension (IIH) is unclear and there is uncertainty on how and when to treat visual complications. A casenote review was performed to examine management of patients with IIH (as per modified Dandy criteria), between 1991 and 1995 at the Royal Victoria Hospital. Forty three patients were identified corresponding to an average annual incidence rate per 100000 of 0.6 for the total and 0.9 for the female population. Casenotes in full were available for 38 patients $(88 \%)$. Almost two thirds had presented to the ophthalmology department, the most commonly reported symptoms being headache $(81 \%)$, transien visual obscurations $(65 \%)$, and sustained visual loss (34\%). Analgesia regularly prescribed in $63 \%$ of patients was effective in none, acetazolamide produced symptomatic improvement in $44 \%$, and CSF opening pressure improvement in $22 \%$. Repeated lumbar puncture, although offering acute symptomatic relief $(58 \%)$, did not prevent visual deterioration. Seven patients $(18 \%)$ underwent thecoperitoneal shunting to preserve vision or to contro headache. Visual acuity deteriorated in two $(5 \%)$, visual fields in seven $(18 \%)$, and two (5\%) developed optic atrophy despite therapy. Almost two thirds were solely followed up by neurologists, only $50 \%$ having more than one formal visual field 
assessment in the year after diagnosis. Visual functioning as measured by visual fields and visual acuity, rather than by repeated CSF pressure monitoring, should guide therapy. Until IIH is better understood and controlled, and trials analysing treatment options are available, coordinated neurological and ophthalmological follow up is mandatory.

\section{INVASIVE MENINGOCOCCAL DISEASE IN} PLYMOUTH

T Ahmed, AY Al-Memar, D Dance, $\mathbf{R}$ Hopkins, S Harrison, I Bray, DC Thrush, Derriford Hospital and University of Plymouth, Plymouth, UK

A retrospective review is presented of 128 patients (age range 4 weeks -88 years), found after a comprehensive search, who presented to Plymouth Hospitals from 1990-5. Four clinical groups were identified: meningitis $(27 \%)$, septicaemia $(30 \%)$, mixed $(34 \%)$, and benign meningococcaemia $(9 \%)$. The "true" mean incidence was $4 \cdot 8 / 100000$ compared with $2 \cdot 9 / 100000$ nationally. Fifteen deaths occurred (crude fatality rate $8 \%$ ). Case fatality rate varied significantly by clinical group $(P=0.029$, Fischer's exact test); the highest being associated with septicaemia (25\%). Several clinical and laboratory variables significantly predicted mortality. $27 \%$ of patients received parenteral antibiotics before admission Treatment was significantly more likely if the patient had a rash $(P=0.0001)$ Prehospital parenteral antibiotics made isolation of meningococcus significantly less likely from blood but did not effect other cultures. Patients taking oral antibiotics were less likely to have a rash on admission ( $P=$ 0.02 ) and the onset to admission interval was longer $(P=0.014)$. The median time from admission to the administration of parenteral antibiotics in hospital was 60 minutes even if a haemorrhagic rash was present. In six patients the initial CSF examination was normal but later proved to be culture positive. $88 \%$ of cases were contact traced by the Department of Public Health Medicine ( $66 \%$ within 24 hours) but the notification rate was only $34 \%$.

TEMPORAL DYNAMICS OF ATTENTION IN VISUOSPATIAL NEGLECT

M Husain, K Shapiro, J Martin, C Kennard, Charing Cross Hospital, London and University of Wales, Bangor, UK

Identification of a briefly presented visual stimulus is associated with an impairment in the ability of normal subjects to detect a second stimulus if it appears within $500 \mathrm{~ms}$ of the first. This has been termed the attentional blink or dwell time.

Patients with unilateral visual neglect are considered to have a disorder of spatial attention. We examined the temporal dynamics of attention in eight stroke patients with left sided visual neglect.

Subjects viewed a rapid sequence of letters presented successively at the same location. All the letters were black except one which was white. The white letter was preceded by 7-15 letters and followed by a sequence of 10 letters. On half the trials, a black $\mathrm{X}$ appeared at some point (varying randomly from trial to trial) in the sequence following the white letter. On the other half of trials, there was no $\mathrm{X}$.

When patients correctly identified the white letter their ability to detect a subsequent $\mathrm{X}$ was significantly impaired for $>1600 \mathrm{~ms}$ afterwards. Age-matched control subjects showed a normal attentional blink which was significantly less protracted and less profound than patients.

This investigation demonstrates an impairment of temporal components of attention in visuospatial neglect.

DIFFERENCES IN DECISION MAKING BETWEEN UNITED KINGDOM AND UNITED STATES NEUROLOGISTS

PJ Martin, B Vickrey, D Gifford, S Delrahim, T Belin, D Smith, DW Chadwick, Walton Centre for Neurology and Neurosurgery, Liverpool, UK

Understanding what factors influence clinical decision making has implications for quality of care and resource management. A survey of United Kingdom and United States neurologists was undertaken to explore variations in practice style and possible influential factors. The questionnaire comprised two sections: (a) evaluation of three clinical scenarios (single seizure, parkinson's disease (PD), dementia); (b) assessment of uncertainty, reliance on investigations, discussion with colleagues and patients, and cost consciousness. 133 consultant $\mathrm{ABN}$ and 550 AAN members responded $(63 \%$ and $92 \%$, respectively).

CT and EEG were considered adequate tests for a single seizure by $94 \%$ of UK responders versus $27 \%$ of US responders ( $P$ $<0.01)$. For the PD scenario $38 \%$ of UK neurologists thought CNS imaging necessary versus $62 \%$ of US neurologists (P $<0.001)$. More US than UK neurologists would start PD medication ( $83 \% v 75 \%$, P $<0.05)$. Conversely nearly all US and UK responders requested CNS imaging for dementia ( $P>0.05)$. Separate multivariate analyses of the PD scenario showed that being a UK neurologist, a lower patient volume, less time in discussion with colleagues, certainty over diagnosis, cost consciousness, and less reliance on tests were all associated with not ordering investigations ( $P$ values $<0.05)$. Overall, UK and US neurologists were equally cost conscious $(P=0 \cdot 84)$. The UK neurologists were less anxious about uncertainty, less reliant on investigations, less reluctant to discuss uncertainty with colleagues but more reluctant to share uncertainty with patients ( $P$ values $<0.001$ ) .

Significant differences in UK and US perception and practice are apparent with US neurologists being more likely to perform tests in two scenarios and start treatments in one. Diagnostic certainty, cost consciousness, and test reliance influenced decision-making in both groups.

\section{TREMOR ASSOCIATED WITH BENIGN IGM} PARAPROTEINAEMIC NEUROPATHY

PG Bain, T Britton, I Jenkins, J Rothwell, P Thompson, PK Thomas, D Brooks, CD Marsden, Institute of Neurology, London, UK

The clinical and neurophysiological features of six patients with action tremor of the upper limbs associated with $\operatorname{IgM}$ paraproteinaemic neuropathy are described. Symptomatic tremor was confined to the upper limbs and was broadly symmetric. The frequency of associated rhythmic muscle activity ranged from $2 \cdot 8-5 \cdot 5 \mathrm{~Hz}$ in abductor pollicis brevis and from $3 \cdot 7-5 \cdot 5 \mathrm{~Hz}$ in the forearm flexor muscles. Magnetic brain stimulation, somatosensory evoked potentials, and stretch reflex studies did not provide evidence for delayed conduction within central pathways. Forearm stretch reflexes were present but their latencies were prolonged. Somatosensory evoked potentials were obtained in most patients but were delayed. Wrist tremor could be modulated by mechanical perturbations or median nerve electrical shocks. Simple voluntary wrist movements were of normal duration and peak velocity, but the kinematic profile was asymmetric. Each movement was associated with a triphasic EMG pattern in agonist-antagonist-agonist muscles but the durations of the bursts were prolonged and the onset of the second agonist burst delayed. These results support the hypothesis that distorted, mistimed peripheral inputs reach a central processor (probably the cerebellum) which although intact is misled into producing tremor in certain parts of the body.

SOD-1 MUTATIONS IN UNITED KINGDOM FAMILIES WITH AMYOTROPHIC LATERAL SCLEROSIS

RW Orrell, FA Bowe, RA Hallewell, S Marklund, RJM Lane, J de Belleroche, Charing Cross Hospital, London, Imperial College, London, UK and Umea University Hospital, Sweden

Seventy one United Kingdom families with more than one member affected by amyotrophic lateral sclerosis (ALS) were investigated for mutations of the gene for intracellular copper-zinc superoxide dismutase (SOD-1). Mutations were identified in 14 families (20\%), comprising 10 different missense point mutations in eight codons, and a novel single insertion mutation leading to a frameshift and predicted truncation of the transcribed protein. These were all heterozygote mutations, with autosomal dominant inheritance. In all patients tested, the mutations were associated with a decrease in erythrocyte SOD enzyme activity of between $32 \%$ and $64 \%$ of normal. To determine the influence of these mutations on the development and course of the disease in these families, we further investigated the association of specific SOD-1 gene mutations, and the reduction of SOD enzyme activity, with the clinical features of the patients (including age at onset, disease duration, and clinical presentation). Generally a specific mutation did not define a distinctive pattern in individual patients, either within or between families. This imprecise correlation of mutation with disease phenotype for the subject is of importance when advising patients and families on the investigation of SOD-1 mutations. The finding of these mutations provides insight into the biochemical disturbances underlying ALS, which may influence the development and application of therapies.

ICTAL OROALIMENTARY AUTOMATISMS WITH PRESERVED CONSCIOUSNESS

G Alarcon, RCD Elwes, CE Polkey, CD Binnie, The Maudsley and King's College Hospitals, London, UK

Loss of consciousness and ictal automatisms are considered major clinical manifestations of complex partial seizures. A video tape showing clinical and electroencephal- 
ographic manifestations of a seizure presenting automatisms and preserved consciousness is reported.

A 29 year old man without family history of epilepsy was referred for surgical treatment of epilepsy. At 15 he began to have seizures, starting with an epigastric aura, occasionally developing automatisms (lip smacking, chewing), sometimes followed by tonic-clonic convulsions. At the time of referral he averaged six convulsive seizures a year and one non-convulsive seizure a week. His sleep EEG showed sharpened slow activity over the right anterior quadrant. MRI showed a benign lesion in the mesial aspect of the right occipital lobe. Simultaneous video monitoring and intracranial EEG with subdural strips recording from the right temporal and occipital lobes were undertaken. During one seizure he had pronounced oroalimentary automatisms while holding a conversation with a technician, answering her questions, and explaining details of his seizures. Memory of this event was preserved. At seizure onset spike activity was seen at the mesial occipital strips. At mid-seizure, high voltage sharpened delta was seen throughout the right hemisphere. Left sided scalp electrodes remained relatively uninvolved. The lesion, a dysembryoplastic neuroepithelial tumour, was removed. Surgery was followed by abolition of seizures described above.

As it is agreed that complex partial seizures require impaired consciousness, a history of automatisms with retained consciousness usually suggests non-epileptic attacks. The present case suggests that automatisms in epileptic seizures can take place with minimal loss of consciousness, particularly if there is widespread but unilateral involvement. The need for a revision of the International Classification is suggested.

THE GENETICS OF CREUTZFELDT-JAKOB DISEASE IN THE UNITED KINGDOM MD Zeidler, K Estibeiro, RG Will, CJD Surveillance Unit, Western General Hospital, Edinburgh, UK

The surveillance of Creutzfeldt-Jakob disease (CJD) was reinstituted in the United Kingdom in May 1990. Suspected cases and their family are interviewed and, if informed consent is obtained, blood is taken for prion protein gene analysis. Genetic information is available for 143 of the 276 definite or probable cases identified (including nine of the 10 recently recognised new variant). Twenty two cases were familial, including eight with Gerstmann-Sträussler-Scheinker syndrome and two with fatal familial insomnia. Mutations of the prion protein gene have been identified at codons 102 , $117,178,200$, and 144 base pair insertions. The common polymorphism coding for either methionine or valine at codon 129 of the open reading frame of the prion protein gene is known for 121 sporadic cases. Ninety six were methionine homozygotes, 17 valine homozygotes, and eight methionine/valine heterozygotes. The excess of methionine homozygotes $(79 \% v 37 \%$ of white controls) concurs with previous studies, demonstrating the increased frequency of this genotype in sporadic CJD. All nine patients with the new variant, for whom genetic information is available, are homozygous for methionine at codon 129 . Four of these patients have a family history of dementia but no mutation identified on their $\operatorname{PrP}$ gene, raising the question as to whether other, as yet unidentified, susceptibility genes played a part in the aetiology of their illness.

EXTRAHIPPOCAMPAL CHANGES AND POOR OUTCOME IN TEMPORAL LOBE EPILEPSY SURGERY

S Sisodiya, N Moran, S Free, N Kitchen, J Stevens, W Harkness, D Fish, SD Shorvon, Institute of Neurology, London, UK

Surgery for temporal lobe epilepsy usually discloses hippocampal sclerosis. Postoperatively, $30 \%$ of patients still have seizures. Perioperative complications and incomplete resection do not account for all failures: epileptogenic tissue must persist, although not apparent on routine inspection of preoperative MRI. The existence of dual pathology has recently been re-emphasised; widespread quantitative cerebral abnormalities on MRI commonly accompany apparently focal cerebral dysgenesis. Similar changes probably occur in association with hippocampal disease, accounting for surgical failure despite complete hippocampal excision on postoperative MRI.

Preoperative MRI from 33 controls and 27 patients, comparable in age and sex, with proved hippocampal sclerosis were quantified to examine regional distribution of grey and white matter proportions, enabling identification of subtle structural abnormalities. These were correlated with surgical outcome (mean follow up $30 \cdot 7$ months).

Twelve patients were not seizure free. The presence of quantitative abnormalities was significantly associated with a poor outcome by comparison with outcome in patients without such changes $\left(\chi^{2}, \mathrm{P}<\right.$ 0.005).

Widespread, occult extrahippocampal structural changes may explain surgical failure in these patients. Quantification of preoperative MRI may supplement clinical decision making and prognostication in patients with a generally, but not inevitably, favourable outcome from epilepsy surgery; quantitation is applicable to other lesional epilepsy surgery.

SERUM TUMOUR NECROSIS FACTOR

CORRELATES WITH NEUROPHYSIOLOGICAL PARAMETERS FOR PERIPHERAL

DEMYELINATION IN PATIENTS WITH

GUILLAIN-BARRÉ SYNDROME

MK Sharief, DA Ingram, $M$ Swash, The Royal London Hospital, London, UK

The Guillain-Barré syndrome (GBS) is an acute inflammatory demyelinating polyradiculoneuropathy of unknown cause. Present evidence suggests that autoimmune damage of peripheral nerves, mediated by activated $T$ lymphocytes and macrophages, underlies the pathogenesis of this disorder. Both cell types secrete tumour necrosis factor- $\alpha$ (TNF- $\alpha$ ), a cytokine that exerts well recognised toxic effects on myelin, Schwann cells, and endothelial cells. Increased serum concentrations of TNF- $\alpha$ have been reported in patients with GBS; however, these concentrations may reflect the degree of immune activation rather than a direct pathogenic effect. The constraints of biopsy or necropsy procedures in patients with GBS invariably restrict evaluation of the extent of peripheral nerve damage caused by
TNF- $\alpha$. Therefore, we compared serum concentrations of TNF- $\alpha$ with well established quantitative neurophysiological criteria for acute demyelination in 16 patients with GBS to assess the pathological relation between this cytokine and peripheral myelin damage. We also measured serum concentrations of interleukin (IL)-1 $\beta$ and soluble IL-2 receptor as controls. High serum concentrations of TNF- $\alpha$ were associated with low amplitude of distal compound muscle action potentials from median, ulnar, and common peroneal nerves, and with absent or prolonged F-wave responses. Serum TNF- $\alpha$ concentrations also correlated with delayed distal motor latencies and motor conduction velocities. No significant correlation was found between neurophysiological parameters for demyelination and serum concentrations of IL-1 $\beta$ or soluble IL-2 receptor. These findings provide evidence for a direct role of TNF- $\alpha$ in the pathogenesis of peripheral demyelination in GBS.

THE INTERNATIONAL STROKE TRIAL.

PRELIMINARY RESULTS PART I: EFFECTS OF

ASPIRIN AND HEPARIN SEPARATELY AND IN

COMBINATION

PAG Sandercock on behalf of the International Stroke Trial Collaborative Group, University of Edinburgh, Edinburgh, UK

The International Stroke Trial is a controlled trial of antithrombotic therapy in acute stroke, in which patients are randomly allocated (in a factorial design) to: aspirin (300 mg daily) $v$ "avoid aspirin"; unfractionated heparin (12 500 units subcutaneously (sc)) twice daily (bd) $v 5000$ units sc bd $v$ "avoid heparin". Treatment is for 14 days or until hospital discharge if sooner. Recruitment will close by the end of May 1996 (target 20000 patients). By 8 February 199617492 patients had been randomised. Results by allocated treatment are presented for the following events (frequency of events within 14 days among the 16120 patients with data are given): deaths $1489(9 \%)$, recurrent stroke due to ischaemia $606(4 \%)$, recurrent stroke due to intracranial haemorrhage $131(1 \%)$, pulmonary embolism 109 (1\%). Among 13 069 patients with six month data: 8319 $(64 \%)$ were dead or dependent, 2580 $(15.3 \%)$ were independent but not fully recovered, and $2002(15 \cdot 3 \%)$ considered they had made a complete recovery from their stroke. Functional status is not yet known for 168 survivors.

THE INTERNATIONAL STROKE TRIAL. PRELIMINARY RESULTS PART II: OUTCOME AMONG PATIENTS WITH DIFFERING LEVELS OF BASELINE RISK

$C$ Counsell on behalf of the International Stroke Trial Collaborative Group, University of Edinburgh, Edinburgh, UK

The International Stroke Trial is a controlled trial of antithrombotic therapy in acute stroke (methods described in part I). The proportion of patients with each of the following characteristics at entry to the study among the 17492 patients randomised by 8 February 1996 were: age $>75$ years $(44 \%)$; drowsy or coma $(23 \%)$; atrial fibrillation (16\%); lacunar syndrome (24\%); randomised within 12 hours of onset $(37 \%)$; CT performed before randomisation $(67 \%)$. 
Analysis of 14 day case fatality (CFR) among 16120 patients with 14 day data showed substantial variation. In patients with each factor, the 14 day CFR was (\%): coma $(58 \cdot 8 \%)$, drowsy (22.7\%), alert $(4.5 \%)$; age $>80(14 \cdot 1 \%), 70-80(9 \cdot 4 \%)$, 60-70 (6.7\%), $50-60(4 \cdot 3 \%),<50(3 \cdot 4 \%)$; atrial fibrillation present $(17 \cdot 4 \%)$, absent $(7 \cdot 5 \%)$; total anterior circulation syndrome $(18.9 \%)$; partial anterior circulation syndrome $(7 \cdot 7 \%)$; posterior circulation syndrome $(9 \cdot 2 \%)$; lacunar syndrome $(2.3 \%)$; CT infarct visible $(11.4 \%)$; no infarct $(7 \cdot 9 \%)$; CT not done $(8 \cdot 7 \%)$; time to randomisation in hours < $3(13.1 \%), 3-6$ $(12.5 \%), \quad 6-12(10.4 \%), \quad 12-24(8 \cdot 9 \%)$, $>24(7 \cdot 1 \%)$. Results by allocated treatment among patients with each of these different levels of baseline risk will be reported.

PATHOGENESIS OF ISCHAEMIC STROKE ASSOCIATED WITH SYMPTOMATIC EXTRACRANIAL CAROTID ATHEROMA

PM Rothwell, R Villagra, R Donders, CP Warlow, Western General Hospital, Edinburgh, UK

Background-Carotid territory ischaemic stroke is often associated with atherosclerotic narrowing at the origin of the ipsilateral internal carotid artery. The risk of stroke increases with the degree of stenosis, and is reduced after endarterectomy, but the mechanism by which carotid atheroma causes stroke is unclear. Cerebral infarction may result from the reduced cerebral perfusion which occurs distal to a tight carotid stenosis or occlusion, but analogy with coronary atherosclerosis would suggest that plaque instability, rupture, local thrombus formation, and distal embolism may be more important.

Methods-To determine the relative importance of plaque morphology, surface thrombus formation, severity of stenosis, and reduced cerebral perfusion in the pathogenesis of cerebral infarction, the angiographic characteristics of 3007 recently symptomatic carotid plaques were studied, 1739 of which were subsequently examined at endarterectomy in the European Carotid Surgery Trial patients.

Results-The risk of stroke on medical treatment ipsilateral to a recently symptomatic carotid stenosis fell rapidly with time, and was only related to the degree of stenosis for two years after study entry. Categorisation of the plaque surface as smooth or irregular by two independent observers on a consecutive sample of 1001 angiograms was reproducible $(k=0.56$ $95 \%$ CI $0.53-0.59)$. The frequencies of both angiographic plaque surface irregularity on 3007 angiograms and surface thrombus at endarterectomy on 1739 plaques increased with degree of stenosis (both $P<0.0001$ ). Angiographic irregularity was, however, independently predictive of surface thrombus formation after correction for severity of stenosis (risk ratio $(R R)=1.32$, $95 \%$ CI $1 \cdot 16-1 \cdot 44, P=0 \cdot 0006)$. Plaque irregularity was an independent predictor of ipsilateral ischaemic stroke on medical treatment $(\mathrm{RR}=1 \cdot 70,95 \% \mathrm{CI} 1 \cdot 12-2 \cdot 59, \mathrm{P}$ $<0.01$ ), but not the risk of stroke due to endarterectomy or the "background" stroke risk after endarterectomy. The risk of ischaemic stroke on medical treatment distal to a haemodynamically significant internal carotid stenosis ( $>50 \%$ linear stenosis) was not increased in association with stenosis or occlusion of the contralateral internal carotid artery, the ipsilateral external carotid artery, or with collapse of the internal carotid artery distal to the stenosis, and was reduced in patients with usual diastolic blood pressure $<80 \mathrm{~mm} \mathrm{Hg} \quad(R R=0.51$, 95\% CI 0.24-0.97, P<0.05).

Conclusions-Angiographic plaque irregularity is associated with surface thrombus formation, and an increased risk of ipsilateral ischaemic stroke on medical treatment at all degrees of stenosis. The increase in stroke risk with severity of stenosis is at least partly accounted for by the parallel increase in plaque surface irregularity and thrombus formation, but is unrelated to factors likely to reduce cerebral perfusion. These data suggest that embolism from irregular carotid plaques is important in the pathogenesis of ischaemic stroke. The results have important therapeutic implications.

MULTIMODAL IMAGING IN ACUTE STROKE

AR Kenton, AR Moody, PJ Martin, RJ Abbott, Leicester Royal Infirmary, Leicester, UK

Background-The basic data from a multimodal imaging study on acute cerebral infarction (ACI) is reported.

Methods-Twenty nine patients with ACI underwent the following investigations within 24 hours of stroke onset: perfusion MRI, diffusion weighted imaging (DWI), MR angiography, transcranial colour coded sonography (TCCS), carotid ultrasound, and SPECT. Perfusion MRI, DWI, and TCCS are then repeated on day 2 , day 7 , and at three months. Patients were assessed clinically using the National Institute of Health stroke scale and the Barthel Index.

Results-TCCS and MRA yielded very similar results regarding the patency of the basal vessels. Middle cerebral artery (MCA) velocity and MRA are both accurate predictors of three month clinical outcome. MCA velocity on the unaffected hemisphere is also highly correlated with cerebral perfusion, measured both by perfusion MRI and SPECT. Perfusion MRI does not yield accurate prognostic data, but does provide information regarding collateral circulation. DWI shows areas of infarction earlier than standard T2 MR imaging and can be used to measure regions of evolving infarction.

Discussion-Multimodal imaging yields valuable information regarding the eventual clinical outcome of patients. It also allows the comprehensive study of the natural history of the haemodynamic changes that occur both in the macrocirculation (using TCCS and MRA) and the microcirculation (using perfusion MRI and SPECT). These techniques may also have a part to play in selecting and following up patients in therapeutic trials.

VIRAL INFECTION AND DISEASE ACTIVITY IN MULTIPLE SCLEROSIS

S Edwards, M Zvartau, W Irving, H Clarke, L Blumhardt, University of Nottingham, Nottingham, UK

Viral upper respiratory tract infections (URTIs) are a significant risk factor for clinical exacerbations of multiple sclerosis (MS). However, clinical attack rate is $5-10$ times less sensitive as an index of disease activity than gadolinium (Gd-DTPA) enhanced MRI.

In a prospective study of 41 patients with definite MS, all of whom were participating in a randomised, placebo controlled trial of interferon $\beta-1 \mathrm{a}$, the relation was investigated between URTI and disease activity as indexed both by clinical attack rate and by the number and volume of GD-DTPA lesions on MRI. "At risk" periods were defined around symptomatic infections noted in a diary and also around serologically proved viral exposures.

Raised antibody titres were associated with a 3.6-fold increase in MS attacks ( $P<$ $0.006)$, compared to "not at risk" periods (annual attack rate $1 \cdot 6$ ). In at risk periods associated with symptomatic viral infections there was a twofold increase $(P=0.004)$.

The volume of Gd-DTPA enhancement on MRI was not increased during symptomatic infections, but was more than four times greater on scans associated with a rise in antibody titres $(P=0.0004)$ than on scans during periods not at risk.

These results confirm the previously reported association between viral infections and exacerbations of MS and, in addition, suggest a relation between viral induced immune stimulation and the inflammatory reaction across the blood brain barrier.

MANAGEMENT OF SLEEP RELATED BREATHING DISORDERS IN THE SHY-DRAGER SYNDROME J Harcourt, P Spraggs, CJ Mathias, GB Brooks, The National Hospital for Neurology and Neurosurgery, London, UK

Respiratory abnormalities and in particular sleep related breathing disorders are a well recognised feature of the Shy-Drager syndrome (SDS). The management of 18 such patients is reported. Three patients had originally presented with sleep disturbance. Vocal cord movement was graded by laryngoscopy as normal, mild weakness of abduction, or near paralysis. Sleep studies involving oximetry and observation were performed. Obstructive apnoea was detected in six patients, one in combination with mild central apnoea, which was also detected alone in one other patient. Specific treatment of the central apnoeas was considered unnecessary. Many patients with near paralysis of vocal cord abduction had normal sleep studies. There was no relation between the clinical subtypes of the condition (parkinsonian, cerebellar, or mixed) and the nocturnal breathing. In two cases continuous positive airway pressure (CPAP) was used successfully. Surgical treatment was used in four patients. Improving the nasal airway was effective in one patient and an arytenoidectomy with or without a covering tracheostomy was performed on three others. This reversed the airway obstruction in two patients without appreciable dysphonia.

Patients with SDS are at risk of developing sleep related breathing disorders and both medical and surgical treatments may be of value.

MEDIAL PALLIDOTOMY FOR THE TREATMENT OF PARKINSON'S DISEASE: A CONSECUTIVE SERIES OF 26 PATIENTS

RP Gregory, MV Lambert, DGC Rogers, IT Ferguson, SS Gill, Frenchay Hospital, Bristol, UK

Twenty six patients with Parkinson's disease have undergone medial pallidotomy at Frenchay Hospital since 1994. A total of 32 pallidal lesions were made with two additional thalamatomies. Intraoperative neurophysiological monitoring was not used. 
Patient mean age was 60 (range 39-75) years, disease duration 12 years (range 4-30), and Hoehn and Yahr grade 3-4. Medical therapy had been exhausted. Patients were prospectively followed up for a mean of eight (range 3-20) months and were assessed independently of the surgeon (SSG).

All patients had a significant reduction in drug induced dyskinesia. Tremor also responded, but less predictably. Mean disability rating scales improved by $30 \%$ when 'off', but were unchanged when "on". Twenty two patients had a significan increase in overall "on time". Dopaminergic medication was increased in seven, and reduced in four. Three patients had a temporary hemiparesis, two non-disabling field defects, and five permanent worsening of their dysarthria. This was severe in one after simultaneous bilateral procedures, but overall function was improved. No cerebral haemorrhages occurred.

This initial pilot study suggests that medial pallidotomy is an effective treatment for some patients with advanced Parkinson's disease and has an acceptable level of morbidity.

THREE MONTH FOLLOW UP OF UNILATERAL POSTEROVENTRAL PALLIDOTOMY FOR PARKINSON'S DISEASE

M Samuel, V Soland, K Sieradzan, DGT Thomas, DJ Brooks, CD Marsden, NP Quinn, Royal Postgraduate Medical School and Institute of Neurology, London, UK

An initial series of 10 patients with Parkinson's Disease (PD) (mean age 55.6 (8.9) years, mean duration of PD 18.4 (4.8) years, mean "off' modified Hoehn and Yahr stage $3.9(0.6)$ underwent left posteroventral pallidotomy (PVP) because of severe motor fluctuations and levodopa induced dyskinesiae. Pallidotomies were performed under stereotaxic CT and electrophysiological recording guidance. Patients were examined three months pre and three months postoperatively according to the CAPIT protocol. Eight patients have currently undergone follow up. Pre and postoperative median scores were compared by Wilcoxon sign rank tests.

Contralaterally, "off" hemibody motor UPDRS subset scores decreased by $28.6 \%$ $(P=0.02)$, rigidity by $31.3 \% \quad(P=0.01)$ tremor by $75.0 \% \quad(P=0.02), \quad$ and bradykinesia by $14.0 \% \quad(P=0.31)$ Ipsilaterally, "off" rigidity decreased by $30.0 \% \quad(P=0.09)$. "Off" speech, freezing, and gait/postural stability scores did not change significantly. "Off" CAPIT tapping times $(\mathrm{n}=7)$ decreased by $35.0 \%$ contralaterally $(P=0.03)$ and by $18.4 \%$ ipsilaterally $(P=0.09)$. Sit-stand-walk times $(n=4)$ decreased by $38.1 \% \quad(P=0.07)$ There were no significant differences in "on" subset scores. Dyskinesiae were reduced by $58.3 \%$ contralaterally $(P=0.01)$ and $25.0 \%$ ipsilaterally $(P=0.04)$. Complications (out of 10 patients) included one hemineglect, one superficial intracranial haemorrhage, one "off" dysarthria, one transient hemiparesis, and one transient facial palsy.

The principal indication for PVP is disabling contralateral dyskinesia, which improved considerably in this series. The effect on underlying parkinsonism is les dramatic and more variable. Two of the 10 patients (not included in the three month follow up) had persisting motor deficits, but both ultimately considered themselves improved overall. The anticipated antidyskinetic effect of PVP must be set against potential risk of morbidity.

NON-SPECIALIST DIAGNOSIS OF TRANSIENT ISCHAEMIC ATTACK AND MINOR STROKE G Young, PJ Martin, TP Enevoldson, PRD Humphrey, Walton Centre for Neurology and Neurosurgery, Liverpool, UK

Accurate diagnosis of transient ischaemic attack (TIA) and stroke has importan implications for prognosis and secondary prevention.

The referral diagnoses of 500 patients assessed in the cerebrovascular clinic between March and October 1995 were prospectively analysed to determine the accuracy of "non-specialist" diagnosis of stroke and TIA compared with two consultant neurologists with a specialist neurovascular interest.

265 male and 235 female patients (median age 63, range 24-95 years) were seen. $45 \%$ were referred by general physicians, $25 \%$ by GPs, and the remainde by neurologists (18\%), vascular surgeons $(5 \%)$, ophthalmologists $(5 \%)$, and others (3\%).

428 patients were referred with variably localised TIAs or strokes with which there was agreement in 321 cases (75\%). Of 125 carotid stroke and 207 carotid TIA referrals there was agreement in significantly more stroke $(n=97(78 \%))$ than TIA patients ( $=123(59 \%)) \quad(P<0.05)$. Vertebrobasilar TIA/CVA $(n=13)$, migraine $(n=8)$, epilepsy $(n=5)$, hyperventilation $(n=5)$, multiple sclerosis $(n=2)$, and hemiparkinsonism $(n=1)$ accounted for some of the discrepancy. Overall the symptoms of 61 patients $(12 \%)$ could not be diagnosed and the diagnosis of cerebrovascular disease in 107 patients (21\%) was "undone". Alternative causes were found for the symptoms of four patients with asymptomatic carotid stenosis who may otherwise have been inappropriately subjected to carotid endarterectomy.

Non-specialists are significantly more accurate in diagnosing stroke than TIA. It is easy to label an episodic neurologica disturbance as a "TIA" and much effort was expended undoing such diagnoses.

HOME THERAPY WITH SELF INFUSED INTRAVENOUS IMMUNOGLOBULIN IN THE TREATMENT OF ACQUIRED CHRONIC DEMYELINATING NEUROPATHIES

WAC Sewell, N Brennan, M Donaghy, HM Chapel, Churchill Hospital, and Radcliffe Infirmary, Oxford, UK

Objectives-To investigate the feasibility of using high dose $(2 \mathrm{~g} / \mathrm{kg})$ intravenous immunoglobulin (IVIg) for patients with acquired chronic demyelinating neuropathies (CIDP) to self infuse at home.

Methods-Five patients with various chronic inflammatory demyelinating polyneuropathies responsive to IVIg underwent formal training for self infusion of IVIg at home. Each patient underwent an 18 week (six infusion) home therapy training programme, and was assessed as being competent in all aspects of the administration of IVIg, including aseptic reconstitution of the product, venepuncture, and blood sampling, before starting infusions at home. The side effects and safety profile of IVIg, recognition of, and action to take in the event of an adverse reaction were fully discussed. The functional abilities of each patient were self assessed using predefined scales, and recorded in daily diaries. The dose and timing of IVIg was then tailored to each patient using the functional strength recordings in the diaries as a guide.

Results-All five patients experienced considerable improvement in functional ability while on home therapy compared with hospital treatment. There was less fluctuation in strength, and no fade off in power before the next infusion.

Conclusions-Home therapy with IVIg is a feasible and effective technique, well liked by patients, and is associated with excellent control of symptoms in CIDP.

FAMILIAL CRAMP WITH A MUTATION OF THE ADULT SKELETAL MUSCLE SODIUM CHANNEL $\alpha$ SUBUNIT GENE: SODIUM CHANNEL MYOTONIA

RW Orrell, F Lehmann-Horn, RJM Lane, Charing Cross Hospital, London, UK and University of Ulm, Germany

A family with cramps affecting four generations, which had been known within the family as the "family cramp" is described. Medical attention was not sought until a 22 year old student presented with difficulty writing for prolonged periods due to a cramping sensation in the arm and hand. Physical examination showed mild eyelid myotonia and percussion myotonia in the hands, together with electrophysiological myotonia. Her mother had symptoms of cramps especially in the legs and eyes with clinical myotonia of the hands. There was no muscle weakness. The symptoms were exacerbated by cooling and with oral potassium administration ( $80 \mathrm{mmol} \mathrm{KCl}$ ), but there was no muscle weakness. The SCN4A gene was sequenced. This encodes the adult skeletal muscle sodium channel $a$ subunit. The heterozygote point mutation Val1589Met was identified in exon 24. This and similar mutations have been associated with the sodium channel diseasesparamyotonia congenita, hyperkalaemic, and normokalaemic periodic paralyses, and sodium channel myotonia. This phenotype of "stiffness without muscle weakness" is recognised as sodium channel myotonia. It is notable that neighbouring mutations cause the different phenotypes of paramyotonia (with weakness on cooling) and periodic paralysis, which may be determined by the dynamic pathophysiology of the muscle sodium channel.

ASSESSMENT OF SWALLOWING IN THE ACUTE NEUROLOGICAL PATIENT

NP Hinds, TAT Hughes, CM Wiles, University of Wales College of Medicine, Cardiff, UK

Disordered swallowing is common in neurological disorder and may result in pneumonia, dehydration, or malnutrition. The best way for a neurologist to assess swallowing in an acute case is uncertain: previous studies have utilised a qualitative water drinking test. A water test has been used which incorporates a quantitative element and for which there is normative data.

61 patients (34 male), median age 65 (range 17-89), with acute neurological disorder (including 29 with stroke) were investigated prospectively. Swallowing was assessed by a neurologist, independent of the patient's management, within 72 hours 
of admission, using a questionnaire, structured examination and timed water test. Outcome variables included referral to and intervention by a speech therapist, dietary modification, duration of hospital stay, respiratory complications, and death.

$13 / 52(25 \%)$ of assessable patients complained of abnormal swallowing. The water test was abnormal in $22 / 54(41 \%)$ patients, of whom only nine coughed while drinking. 21/54 patients referred to the speech therapist had a median swallowing capacity of $27 \%$ predicted compared with $89 \%$ in those not referred $(P<0.001)$. The sensitivity of an abnormal water test in predicting intervention by the speech therapist was $88 \%$ whereas that based on the complaint of abnormal swallowing was $55 \%$.

It is concluded that assessment using a quantitative water test is a more sensitive indicator of abnormal swallowing requiring intervention than symptoms or a qualitative test alone.

NORMATIVE DATA FOR FUNCTIONS ASSESSED IN PATIENTS WITH NEUROMUSCULAR DISEASE TAT Hughes, CM Wiles, University Hospital of Wales, Cardiff, UK

There is a lack of normative data for interval measures of function used for patients with neuromuscular disease. 181 healthy adults were studied, including a minimum of 10 male and female subjects in each 10 year age band between 15 and 75 years, and $>75$ years. As well as indices of swallowing (previously reported) muscle strength was measured (right elbow extension and hip flexion) using a hand held myometer, walking speed over $6 \mathrm{~m}$ with a turn, and, using a hand held spirometer, the difference in \% predicted forced vital capacity (FVC) between sitting and lying (often used as an index of diaphragmatic function).

Regression analysis showed that age and sex were determinants ( $r^{2}$ adjusted) of the strength of elbow extension (61\%), hip flexion $(72 \%)$, and walking speed $(56 \%$, all $P<0.001)$, but not of change in FVC (mean fall $=4.5 \%(S D 6.9)$ )

These data allow measures at the level of impairment (strength) and disability (swallowing, breathing, and walking) to be expressed in terms of a predicted mean for age and sex; the derived $95 \%$ prediction interval can be used to define abnormality in an individual patient. Ratio indices of function, expressed thus, are a useful adjunct to clinical assessment, particularly in monitoring.

FALLACIES IN THE PATHOLOGICAL

CONFIRMATION OF THE DIAGNOSIS OF ALZHEIMER'S DISEASE

JV Bowler, D Munoz, H Merskey, VC Hachinski, University of Western Ontario, London, Canada, and Charing Cross Hospital, London, UK

Most series report that the necropsy confirmed clinical diagnostic accuracy for Alzheimer's disease (AD) is $80 \%$. This figure contains two fallacies. Firstly, it includes cases in which $\mathrm{AD}$ exists with other diseases affecting cognition, and secondly, it excludes cases without necropsy. Data from The University of Western Ontario Dementia Study were reanalysed to estimate the effect of these errors.

Clinical and pathological diagnoses were made according to standard criteria. The pathological material was interpreted in two ways, allowing and disallowing coexistent disease. In cases not dying, or dying without necropsy, progression of cognitive loss on follow up was used as a marker for degenerative dementia.

Cases of AD numbered $200(65 \%)$, vascular dementia $12(4 \%)$, mixed (vascular dementia with AD) dementia 47 (15\%), and other diagnoses 48 (16\%). 192 cases $(63 \%)$ died and $122(64 \%)$ had necropsies. The positive predictive value of a diagnosis of $\mathrm{AD}$ was $81 \%$ when true positive results included coexistent diseases, falling to $44 \%$ when limited to pure $\mathrm{AD}$. In cases without necropsy, $22 \%$ had no evidence of dementia after prolonged follow up. Allowing for these, the overall diagnostic accuracy fell to $70 \%$ for any $\mathrm{AD}$ and $38 \%$ for pure $\mathrm{AD}$.

The combined fallacies of dual pathology and partialism reduce clinical diagnostic accuracy to $38 \%$ from currently quoted figures of $80 \%$. These errors are greatest in early dementia. This has important implications for treatment, prognosis, and clinical trials.

HEROIN PYROLYSATE

LEUCOENCEPHALOPATHY

AP Claxton, MR Rose, R Greenwood, Homerton Hospital, London, UK

In 1982 a toxic leucoencephalopathy resulting from the inhalation of fumes from heated heroin was described from Holland. Since then, there have been a few othe cases reported, from Italy, Germany, and Spain, but not from the United Kingdom. Patients present with a subacutely progressive cerebellar syndrome. The identity of the toxin responsible remains unknown.

Three cases have been seen recently in the United Kingdom and demonstrate the unusual CT and MRI appearances. These comprise symmetric non-enhancing abnormality with no mass effect, hyperintense on T2 weighted MRI, and limited to the white matter of the cerebellar hemispheres, internal capsules, and corona radiata.

EFFECT OF INTRAVENOUS

METHYLPREDNISOLONE ON LEUCOCYTE AND ENDOTHELIAL CELI ADHESION MOLECULE EXPRESSION IN MULTIPLE SCLEROSIS A Droogan, M Treacy, A Crockard, S Hawkins, Royal Victoria Hospital, Belfast, UK

Intravenous methylprednisolone (IVMP) may inhibit inflammatory cell recruitment to active multiple sclerosis (MS) lesions by decreased leucocyte or endothelial cell adhesion molecule expression. Periphera blood mononuclear cells were isolated from 15 patients with $M S$ in relapse receiving a five day course of IVMP (500 mg/day) and 15 normal subjects. Samples were obtained pretreatment, at six and 24 hours after the first dose, and 48 hours after completion of therapy. Levels of L-selectin, LFA-1, Mac-1 and VLA-4 expression were determined on $\alpha \beta$ and $\gamma \delta \mathrm{T}$ cells and monocytes by dua colour immunofluorescence flow cytometry. Serum levels of L-selectin, E-selectin, ICAM-1 and VCAM-1 were measured by ELISA. Adhesion molecule expression was normal on circulating $T$ cells and monocytes in active MS. IVMP resulted in significant changes in monocyte adhesion molecule expression: increased $L$-selectin expression at 24 hours $(P=0.0002)$, decreased Mac-1 expression at six hours $(P=0.003)$, and decreased VLA-4 expression six hours ( $P=$ $0.005)$ and 24 hours $(P=0.004)$ after treatment. $T$ cell adhesion molecule expression was unaffected. Serum E-selectin was reduced at six hours $(P=0.01)$ and 24 hours $(P=0.0002)$ after treatment. IVMP alters the distribution and kinetics of monocyte adhesion molecule expression and endothelial E-selectin expression, which may limit monocyte recruitment to areas of tissue destruction in MS.

TREATMENT OF MILLER FISHER SYNDROME WITH HUMAN IMMUNOGLOBULIN

M Chowdhury, A Kennedy, D Wren, F Schon, Atkinson Morley's Hospital, London, UK

There is good evidence from randomised controlled trials that human immunoglobulin has a significant benefit in the rate of recovery of Guillain-Barré syndrome. In the related Miller Fisher syndrome (MFS), no such trial data exist. Three recent patients with MFS, treated with human immunoglobulin $(0.4 \mathrm{~g} / \mathrm{kg} /$ day for five days $)$ are described. All three patients were men, two aged 29 and one aged 52 years. Each had a preceding upper respiratory tract infection, and on admission were sufficiently ataxic to be unable to walk. Each had a variable degree of ophthalmoplegia. Each had a normal brain MRI. CSF protein was normal in two patients and raised in one. Nerve conduction studies were normal in two patients and showed a sensory axonal neuropathy in one. Two patients had positive serum antiganglioside antibodies, anti GQ1b, and anti GM1. By the time of discharge, five, eight, and 11 days after starting treatment with human $\gamma$-globulin, all three patients were able to walk fully independently. There was a less dramatic improvement in ophthalmoplegia. In view of the rarity of Miller Fisher syndrome, it is unlikely that there will ever be proper randomised controlled trials to look at the effect of $\gamma$-globulin. A recent review of cases in the world literature, found an average length of time for recovery of function including walking of about 10 weeks, with a range from two weeks to eight months. The recovery of the three patients described here was within 11 days of starting treatment, supporting the use of human immunoglobulin in this unusual condition.

WEIGHT GAIN ON VALPROATE: REDRESSING THE BALANCE

MJ Tomlinson, DJ Easter, CG O'BryanTear, C Verity, Sanofi Winthrop Ltd and Addenbrookes Hospital, Cambridge, UK

Valproate has traditionally been associated with a greater incidence of weight gain than other anticonvulsants. A prospective, randomised three year trial compared valproate ( $\leqslant=30 \mathrm{mg} / \mathrm{kg}$ body weight) and carbamazepine $(\leqslant=20 \mathrm{mg} / \mathrm{kg})$ in 260 children (aged 4-15 years) with newly diagnosed epilepsy. Failure of seizure control/occurrence of adverse events permitted switching to the alternative drug. Any child requiring additional antiepileptic medication was withdrawn from the study. A retrospective analysis of body weight data showed more reports of weight gain as an adverse event in the valproate group than in the carbamazepine group (22 reports in 14 patients versus nine reports in five patients) However, among the 211 patients (103 on valproate and 108 on carbamazepine) in 
whom weight measurements were taken, there were no differences between treatments in mean weight, percentage weight gain from baseline, or incidence of excessive weight velocity. Of eight patients reporting weight gain on valproate and switched to carbamazepine because of poor seizure control and/or adverse events (including weight gain), weight measurements were available in four; three of these continued to gain weight on carbamazepine. Weight gain may be erroneously attributed to valproate and clinicians should consider all possibilities for observed weight gain before changing antiepileptic drugs.

AN INTERNATIONAL COMPARISON OF THE INCIDENCE OF STROKE AND ITS PATHOLOGICAL TYPES

CLM Sudlow, CP Warlow for the International Stroke Incidence Collaboration, Western General Hospital, Edinburgh, UK

Comparing stroke rates in different parts of the world may increase understanding of both aetiology and prevention. The incidence of stroke and its pathological types (cerebral infarction, primary intracerebral haemorrhage, and subarachnoid haemorrhage) were compared in recent studies from around the world. Studies with a mid-year of 1984 or later, fulfilling standard criteria for a comparable, community based study, provided original data for comparative analyses. By mid-1995, data were available from 11 studies in Europe, Russia, Australasia and the United States, comprising 3.5 million person-years and 5575 incident strokes. Age and sex standardised incidence rates for 45 to 84 year olds were similar (between about 300 and 500/100 000/year) for most studies but were significantly lower for Dijon, France (238/100 000/year) and higher for Novosibirsk, Russia (627/100 000/year). In 75 to 84 year olds, however, stroke incidence in Novosibirsk no longer ranked higher than other studies. The distribution of pathological types, when these were reliably distinguished, did not differ between studies. Further work is needed to investigate the potential explanations for the differences, and to extend the growing knowledge of stroke incidence to other parts of the world.

THE PIRIFORMIS SYNDROME: A SCIATIC NERVE ENTRAPMENT

AY Al-Memar, $\mathbf{N}$ Hudson, G Thomas, $P$ Hughes, HSK Wimalaratna, Derriford Hospital, Plymouth, UK

The piriformis syndrome is an uncommon cause of sciatic pain. It is poorly recognised and inadequately described, but is a disabling condition. Fifteen patients (four men), mean age 59.9 (range $32-88$ ) years were studied in detail. The aim of this study was to identify important diagnostic clinical and neurophysiological features of this syndrome. MRI of the gluteal region was done to reveal a possible pathology. Some of the patients have already undergone decompression surgery with complete relief of symptoms. The following clinical and neurophysiological features were found to be useful in verifying the diagnosis:

Unilateral gluteal pain with or without leg pain.

Localised tenderness over the piriformis muscle.
Aggravation of the symptoms by "Plymouth manoeuvre".

Absent or asymmetry of the ankle jerk.

Sensory abnormalities in the lateral aspect of the foot and the fifth toe.

Absent or asymmetric (low amplitude) sural SNAP.

Prolonged or dispersed tibial nerve $F$ waves ( $>2 \mathrm{~ms}$ ) from the affected side.

Abnormalities in $\mathrm{H}$ reflex.

Normal lower limb nerve conduction velocities.

Denervation potentials in gastrocnemius and/or hamstring muscles.

CT and/or MRI of the lumbosacral region is normal.

In conclusion, a range of clinical and neurophysiological features have been identified which are useful in arriving at a confident diagnosis of piriformis syndrome.

THE LEEDS MSQOL SCALE: A DISEASE SPECIFIC MEASURE OF QUALITY OF LIFE IN MULTIPLE SCLEROSIS

HL Ford, A Tennant, $\mathrm{MH}$ Johnson, St James University Hospital, Leeds, UK

Quality of life measures are increasingly being used as outcome measures in clinical trials. Disease specific measures are more sensitive than generic measures to small, clinically important changes in a patient's status. The development of a disease specific measure of quality of life for people with multiple sclerosis is described.

The items in the scale were selected in focus group sessions with a group of people with multiple sclerosis, thus ensuring face validity for respondents. The scale was designed as a self report questionnaire with a four step Likert scoring system. It was tested in $\mathbf{1 5 0}$ people with multiple sclerosis from a comprehensive prevalent register of people with multiple sclerosis in Leeds, and found to be both internally consistent and valid. Cronbach's $a$ was 0.77 and convergent validity -0.79 (Spearman's rank correlation) when tested against the general wellbeing index. Further aspects of construct validity were tested by fit of the data to the Rasch model. The scale showed adequate fit to the Rasch partial credit model, thus confirming the presence of a single underlying construct of quality of life. The scale was also found to be ordinal in nature, and was able to identify three separate groups of patients along the quality of life construct.

The Leeds MSQoL scale is a 16 item scale which is brief, user friendly and practical to administer as a postal questionnaire.

COGNITIVE FUNCTION IN ATAXIA-

TELANGIECTASIA

IB Colvin, GG Lennox, University of Nottingham, Nottingham, UK

Ataxia-telangiectasia (A-T) is a neurodegenerative disorder associated with immunodeficiency and a predisposition to malignancy. The core neurological features of cerebellar ataxia, oculomotor apraxia, and movement disorder appear clinically to be accompanied by a variable degree of cognitive impairment of frontal type. Therefore a neuropsychological battery was used to study cognition in 18 adult patients with $A-T$ and 12 matched controls with cystic fibrosis.

Patients with A-T scored significantly lower on the Wisconsin card sorting test, a modified Tower of London test and tests of verbal fluency, similarities, and digit span. These results are compatible with our clinical impression of frontal lobe dysfunction.

Patients with A-T scored significantly lower on the visual component of the Warrington recognition memory test and some subtests of the visual object and space perception battery, which may reflect their oculomotor difficulties.

They scored significantly lower on the national adult reading test and arithmetic tests. Interpretation of these abnormalities is confounded by differences in educational experience.

There was no difference in mini mental state examination and hospital anxiety and depression scores.

It is concluded that A-T is associated with an identifiable pattern of cognitive deficit This has important implications for the education, employment, and general care of people with A-T.

VISUOMOTOR INTEGRATION AND THE

PREMOTOR CORTEX IN HUMANS

SR Jackson, M Husain, C Kennard, Charing Cross and Westminster Medical School, London and University of Wales, Bangor, UK

Two important developments in primate evolution are stereopsis and the ability to execute prehensile movements. Studies in non-human primates suggest that the dorsolateral premotor cortex may be primarily involved in the planning of limb movements, whereas ventrolateral premotor cortex may be more directly involved with the on line execution of reaching movements.

In this study a high resolution limb tracking system was used to determine the effect of lesions to the lateral premotor cortex on the planning or on line control of prehension movements in humans. Two patients ( 1 and 2 ) with unilateral lesions due to infarction involving the inferior left premotor cortex were compared with control group of normal subjects on tasks using naturalistic prehension movements. In common with control subjects, both patients were clearly able to scale the velocity of their reaching movements for different movement amplitudes. However, patient 1's reaches were fastest when reaching without visua feedback, whereas patient 2 showed a highly significant reduction in peak velocity when reaching with only monocular visual feedback. Furthermore, both patients exhibited reduced movement durations, and deceleration phases when reaching without visual feedback, and conversely, were especially slowed when reaching with only monocular visual feedback.

These findings seem to confirm that the inferior premotor cortex may participate in the planning or on line execution of visually guided prehension movements in humans.

ANALYSIS OF THE 5 ' TANDEM REPEAT AND EXON SEOUENCES OF THE MYELIN BASIC PROTEIN GENE IN MULTIPLE SCLEROSIS SE Price, J James, C Mason, A Boots, L Hinks, RJ Thompson, Southampton General Hospital, Southampton, UK

An abnormality of myelin basic protein (MBP) or its gene in multiple sclerosis (MS) aetiology is suggested by studies showing linkage or association of the gene with MS. MBP gene disorders cause dysmyelinating diseases in mice and MBP provokes 
experimental allergic encephalomyelitis in animal models of MS.

The MBP gene in patients with MS and controls was studied by two methods. The (TGGA)n repeat upstream of the gene was amplified using the polymerase chain reaction (PCR) and electrophoresis. Five alleles were identified using a primer pair encompassing the entire repeat. No significant difference was found in allele distribution between MS and control groups. However eight alleles identified by a primer pair encompassing a $3^{\prime}$ portion of the repeat showed a difference between MS (n $=41)$ and control $(n=41)$ groups. $\left(\chi^{2}=\right.$ 17.76 (7 df) $P<0.02)$.

The seven coding exons and $5^{\prime}$ and 3 untranslated regions of the MBP gene were investigated using the PCR single strand conformation polymorphism method (MS n $=36$, control $n=36$ ). Three mutations were found in the coding exon. One (in exon 3 ) was conservative but two (exon 1) altered amino acid sequence. There were no mutations in the $5^{\prime}$ but several in the long 3 untranslated regions. No mutations were commoner in MS.

Therefore an association of part of the $5^{\prime}$ tandem repeat with MS was found but no MBP gene abnormality had increased frequency in MS.

A NEW NON-INVASIVE METHOD OF ASSESSING DYNAMIC CEREBRAL AUTOREGULATION RP White, HS Markus, Kings College School of Medicine and Dentistry, London, UK

A new non-invasive method of measuring dynamic as opposed to static cerebrovascular autoregulation may have many applications in cerebrovascular disease. The rate of rise of cerebral blood flow (CBF) velocity measured by transcranial Doppler is compared with that of arterial blood pressure (BP) (monitored via finger plethysmography), after a sudden reduction in $\mathrm{BP}$ induced by hyperaemia after rapid deflation of bilateral thigh cuffs. Curve fitting software computed an autoregulatory index (ARI) from 0-9. Its application is described in carotid stenosis (>70\%). In 19 patients one had no acoustic window and in three a sufficient fall in MAP could not be induced, leaving 15 patients with 20 stenoses. 22 patients with carotid stenoses and 12 normal controls were compared. ARI was compared with vasomotor reactivity to

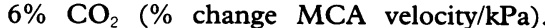
ARI was significantly impaired in the MCA ipsilateral to the carotid stenosis; mean (SD) $6.2(1.2)$ v $2.5(2 \cdot 1)$ (Mann-Whitney U test, $\mathrm{P}<0.0001)$. The method identified a subgroup of patients with carotid stenosis with very impaired ARI (table).

There was a correlation with impaired $\mathrm{CO}_{2}$ reactivity (Spearman $\rho=0.487, \mathrm{P}<$ 0.05 ) but a subgroup with mild impairment of $\mathrm{CO}_{2}$ reactivity showed a severe reduction in ARI.

Dynamic autoregulation is a more sensitive marker of vasomotor impairment than $\mathrm{CO}_{2}$ reactivity. It may allow identification of haemodynamically significant carotid stenosis for endarterectomy

\begin{tabular}{lll}
\hline & Normals & $\begin{array}{l}\text { Carotid } \\
\text { stenosis }\end{array}$ \\
\hline Very impaired ARI $(<1 \cdot 9)$ & 0 & 7 \\
Impaired ARI (2-3.9) & 0 & 9 \\
Normal ARI $(>4)$ & 22 & 4 \\
\hline
\end{tabular}

and identify patients less able to compensate for reduced perfusion pressure after antihypertensive therapy.

MAGNETIC RESONANCE ANGIOGRAPHY IN ACUTE CEREBRAL INFARCTION

AR Kenton, AR Moody, PJ Martin, RJ Abbott, Leicester Royal Infirmary, Leicester, UK

Introduction-As part of an ongoing study of acute cerebral infarction (ACI), magnetic resonance angiography (MRA) was performed on 28 patients to assess its prognostic ability.

Methods - 28 patients with ACI confirmed by MRI underwent MRA within 24 hours of stroke onset. Vessels were classified as either normal attenuated or absent. Patients were assessed clinically at three months using the Barthel index (BI). Stroke subtype was classified according to the Oxford Community Stroke Project.

Results - 10 patients had proximal middle cerebral artery (MCA) occlusion: at three months, four were dead, two required constant nursing care, three were dependent on one carer and one patient was fully independent. All patients had TACI except the last one, who had a PACI. Of the five patients who had either attenuation of the proximal portion of the MCA or MCA branch occlusions, one was dead (TACI), another was in a nursing home (LACI), and three were independent (two PACI one TACI). 14 patients either had attenuation of MCA branch vessels (three TACI, three LACI, one PACI) or a normal scan (six LACI, one PACI). All of these patients were fully independent except one, who required minimal nursing care.

Conclusion-MRA is a simple non-invasive method of imaging the cerebral vasculature. The presence of an occluded MCA is usually associated with a TACI and a poor prognosis. Normal scans or MCA branch attenuation is associated with a more favourable prognosis and patients tend to have either lacunar strokes or PACI. It is the patients with MCA branch occlusions that have an unpredictable prognosis which does not seem to depend on the stroke subtype. Perfusion studies with MRI are currently being assessed in these patients.

AUDIOVESTIBULAR PATHOLOGY IN NEUROSARCOID

PA Savundra, RA Davies, LM Luxon, National Hospital for Neurology and Neurosurgery, London, UK

A series of 12 patients with neurosarcoid (M:F 1:2; mean age 36.6) referred for neuro-otological investigation was analysed. Ten had vertigo, which was the presenting symptom in six. Nine had a peripheral vestibular lesion (one BPPV, one bilateral) of whom one had an asymptomatic complete canal paresis. Four had central vestibular pathology. Eight had a hearing loss, six improving spontaneously, and two after steroids. Three had cochlear nerve pathology, of whom two had facial nerve pathology, one with trigeminal nerve pathology, and one had associated optic nerve pathology. Two presented with hydrocephalus, one with transverse myelitis, two with associated peripheral neuropathy, and three with choroidoretinitis. MRI showed white matter changes in $4 / 5$ patients scanned.

The diagnosis of neurosarcoid is difficult.
The Kveim test was negative in two patients. SACE levels were normal in two patients with raised CSFACE, but TLCO was abnormal in all the patients tested and the biopsy of nasal or skin lesions was positive in the four patients tested.

Audiovestibular pathology is relatively common in neurosarcoid, but can be asymptomatic. It should be identified as the hearing and vestibular pathology may be steroid sensitive.

EFFECTS OF AN ACOUSTIC STARTLE ON

REACTION TIME IN PATIENTS WITH

PARKINSONISM

E Tolosa, F Valldeoriola, F Nobbe, E Muñoz, N Fabregat, J Valls-Sole, Hospital Clinic I Provincial, Barcelona, Spain

In normal subjects, an acoustic startle (AS) given together with the "go" signal in a reaction time paradigm, shortens significantly the reaction time. The neurophysiology of such an effect involves central neural structures that may be functionally impaired in patients with parkinsonian syndromes. The influence of AS on reaction time (RT) was analysed in eight patients with Parkinson's disease (PD), seven patients with progressive supranuclear palsy (PSP), seven patients with multisystem atrophy (MSA), and seven healthy age matched controls. RT was measured as the time from the "go" signal to the closure of a switch and expressed the acceleration as a percentage of the RT in trials without AS. Patients with PD or MSA independently of all clinical variables, presented acceleration of RT with a startling stimulus, delivered randomly. The mean acceleration of $R T$ in trials containing the startling stimulus was $22 \%$ in patients with $\mathrm{PD}, 20 \%$ in patients with MSA. These figures were not different from those obtained in control subjects (28\%). By contrast, acceleration of RT was not seen in patients with PSP $(-0.4 \%)$. We conclude that AS shortens the motor responses of RT in PD and MSA but not in PSP. The pathological substrate of PSP, involving those brainstem areas where the startle reflex is organised, might explain this finding. Startle induced shortening of the reaction time is a neurophysiological tool that can be used to distinguish patients with PSP from those with PD and MSA.

PERIODIC LIMB MOVEMENT DISORDER

AJ Williams, D Kidd, $\mathbf{R}$ Howard, St Thomas' Hospital, London, UK

Periodic limb movements in sleep are common but the resulting disorder (PLMD) is often unrecognised. During 1995 the abnormality was noted in six patients referred for other reasons to this neurological/respiratory unit, and in whom the disorder was clinically relevant. The table summarises the clinical data.

PLMD is reported to present most often with insomnia (particularly difficulty initiating sleep as in patient 2), and less often with hypersomnia (excessive daytime sleepiness). More unusual, however, are the other presentations encountered here such as parasomnia (abnormal movements in sleep as in patient 1) non-specific fatigue (patients 4, 5), or late functional deterioration in old polio (the post polio syndrome patients 3, 6). Notably, all patients had the complaint of restless legs.

It is concluded that presentation of this common disorder may be different in a 
Summary of the clinical data

\begin{tabular}{lllllll}
\hline Patient & Age & Sex & Presentation & $R L S \pm$ & Other diagnosis & NPSG diagnosis \\
\hline 1 & 77 & M & $\begin{array}{l}\text { Abn movements } \\
\text { in sleep }\end{array}$ & + & Polymyositis & PLMD/REMBD \\
2 & 68 & F & $\begin{array}{l}\text { Insomnia } \\
\text { Postpolio } \\
\text { syndrome }\end{array}$ & ++ & $\begin{array}{l}\text { Panhypopituitary } \\
\text { Old polio }\end{array}$ & PLMD \\
3 & 41 & F & + & Fibromyalgia & PLMD $/ a \delta$ \\
4 & 44 & F & $\begin{array}{l}\text { Fatigue } \\
\text { sleep }\end{array}$ \\
5 & 28 & F & $\begin{array}{l}\text { Fatigue } \\
\text { Postpolio } \\
\text { syndrome }\end{array}$ & ++ & Old polio & PLMD \\
\hline
\end{tabular}

NPSG = nocturnal polysomnography; REMBD = REM behaviour disorder; RLS = restless legs.

PLMD was diagnosed by NPSG in five and from the history in one.

selected population. Questions about sleep are important and should include those about restless legs and abnormal movements in sleep.

RELIABILITY, VALIDITY, AND RESPONSIVENESS OF THE KURTZKE EXPANDED DISABILITY STATUS SCALE IN MULTIPLE SCLEROSIS JC Hobart, DL Lamping, JA Freeman, AJ Thompson, Institute of Neurology, London, UK

The Kurtzke expanded disability scale (EDSS) remains the "gold standard" disability measure for patients with multiple sclerosis (MS) and is used extensively in effectiveness studies despite concerns over some of its scientific properties. However, its evaluation in accordance with accepted guidelines has been incomplete. This study examined the reliability, validity, and responsiveness of the EDSS in patients admitted to a rehabilitation unit.

132 patients with MS with moderate and severe disability were studied: mean age $\mathbf{4 2}$ (range 16-73); 64\% female; $75 \%$ secondary progressive disease; mean EDSS $7 \cdot 1$ (range $5 \cdot 0$ to $9 \cdot 0$ ).

For reliability (intraclass correlation coefficient $): \quad$ interrater $=0.77 \quad(n=116)$; intrarater $=0.92(n=32)$.

For validity $(n=66)$ : correlations with six other disability indices rated at the same time (convergent construct validity) ranged from 0.37 (patient report visual analogue scale) to 0.89 (Barthel index). Correlations with six non-disability measures (discriminant construct validity) ranged from 0.02 (short-form 36 change in health dimension) to 0.63 (Waterlow pressure sore risk score).

For responsiveness $(n=64)$ : using pre and postrehabilitation EDSS ratings; effect size $=0.06$.

These results support the EDSS as a reliable and valid disability measure in this restricted population. However, responsiveness is poor which raises concerns over its use in phase III clinical trials.

MIDDLE CEREBRAL ARTERY VELOCITY AS A PROGNOSTIC INDICATOR IN ACUTE STROKE AR Kenton, AR Moody, PJ Martin, RJ Abbott, Leicester Royal Infirmary, Leicester, UK

Introduction-Transcranial colour coded sonography (TCCS) is a non-invasive method of imaging the basal cerebral vessels. 26 patients were studied within 24 hours of acute cerebral infarction (ACI) to see if clinical outcome can be predicted with this technique.

Methods-The basal cerebral arteries were imaged using TCCS and the velocity in the middle cerebral artery (MCA) was measured with pulsed wave Doppler. The side to side difference in MCA velocity (asymmetry index: AI) was calculated according to Zannette. Patients were followed up at three months and assessed clinically using the Barthel index.

Results-Nine patients presented with MCA occlusion, thus giving an AI of $200 \%$; at three months, four were dead, two needed constant nursing care, and three patients relied on one carer (BI median: 15). 11 patients had a normal $\mathrm{AI}$ of $<20 \%$ and all were fully independent at three months (BI median: 100). Four patients had a low AI (range: $24 \%-77 \%$ ); at three months, one patient was dead, one was dependent on one carer (BI median: 75). Two patients had a high AI $(+78 \%,+44 \%)$; one was fully independent at three months, and the other patient was in a nursing home (this patient also had a complete internal carotid artery occlusion). The correlation between $\mathrm{AI}$ and the BI was -0.72 .

Conclusion-Patients with MCA occlusion tend to have a very poor prognosis whereas patients presenting with normal velocities have a favourable outcome. Those patients with reduced MCA velocity have an intermediate prognosis. There is a high correlation between the $\mathrm{AI}$ and the $\mathrm{BI}$ at three months suggesting that TCCS is a useful tool in predicting the eventual clinical outcome in ACI.

\section{AMYLOID $\beta$-PEPTIDES AS NEURITE GROWTH} INHIBITORY MOLECULES

AJ Larner, Department of Anatomy, Cambridge, UK

Objective/methods-To test the hypothesis that amyloid $\beta$-peptides $(\mathrm{A} \beta)$, which accumulate in the plaques of Alzheimer's disease (AD) brain, may act as neurite growth inhibitory molecules, embryonic chicken neurons from both dorsal root ganglia (DRG) and retina were grown in vitro in the presence of synthetic soluble $A \beta$. Neurite outgrowth was measured using a calibrated graticule incorporated into the eyepiece of a Zeiss Axiovert phase contrast microscope.

Results-Neurite outgrowth from both DRG and retina was reduced in the presence of $\beta 25-35$, the presumed neurotoxic moiety of $\beta 1-40$, compared with controls (Student's $t$ test, $\mathrm{P}<0.001$ ), but not in the presence of $\beta 1-40$ or $\beta 1-28(\mathrm{P}>$ 0.5 , both conditions). Serial dilution of $\beta 25-35$ showed that inhibition of neurite outgrowth was first apparent at $10 \mathrm{ng} / \mathrm{ml}$ (about 9.4 $\mathrm{nM}$ ), and that the degree of outgrowth inhibition was concentration dependent. The inhibitory effect of $\beta 25-35$ was partially reversible: switching DRG from growth medium containing $\beta 25-35$ (1 $\mu \mathrm{g} / \mathrm{ml})$ to either standard unsupplemented medium $(\beta / S)$ or freshly made $\beta 25-35-$ supplemented medium $(1 \mu \mathrm{g} / \mathrm{ml} ; \beta / \beta)$ showed significantly greater outgrowth in the first than the second $(P<0.05)$.
Conclusion-Shorter A $\beta$ show neurite growth inhibitory activity in vitro. Because shorter $A \beta(\beta 17-42)$ accumulate in diffuse, but not neuritic, plaques in $\mathrm{AD}$ brain, this activity may explain the differing morphology of these lesions.

AN UNUSUAL CASE OF RECURRENT SYNCOPE SJM Smith, CJ Mathias, R Bannister, The National Hospital for Neurology and Neurosurgery, London, UK

Syncope in young women is usually vasovagal in nature. A case of unusual syncope is reported in an 18 year old woman with a four year history of stereotyped attacks of loss of consciousness (LOC) and postural hypotension while sitting or standing. Standard autonomic function tests were normal. Simultaneous monitoring of EEG, ECG, airflow, intra-arterial pressure, and arterial blood gases was performed. Typical attacks were reproduced after a few seconds of tilt to $45^{\circ}$. The initial clinical change was rapid breathing followed in turn by apnoea, LOC blood pressure (BP) fall (mean supine 98/47 to 57/33) after $10 \mathrm{~s}$, and widespread slow waves in the EEG. Cardiac rate rose from resting $67 \mathrm{bpm}$ to 105 during the attack. Spontaneous recovery occurred while tilted, with return of consciousness, respiratory effort, and rise in BP.

Hyperventilation or the Valsalva manoeuvre did not precipitate an attack. LOC early in the attack had suggested an epileptic basis; however, the interictal EEG was normal and anticonvulsant treatment was ineffective. MRI and IV DSA were normal. It is proposed that this unusual sequence of apnoea, LOC, and systolic BP fall represents a functional form of syncope, with a unique reflex basis, perhaps linked to changes similar to a Valsalva manoeuvre.

REMACEMIDE AND ITS METABOLITE ARL12495 REDUCE SPONTANEOUS SYNAPTIC POTENTIALS IN $\mathrm{MG}^{++}$FREE KREBS; AN IN VITRO SPINAL CORD STUDY

SS Hasan, AE King, PM Crawford, University of Leeds, Leeds and York District Hospital, York, UK

The anticonvulsant remacemide and its metabolite ARL12495 are known to possess NMDA (N-methyl D-aspartic acid) receptor antagonist properties in mammalian brain These compounds have been tested in the rat spinal cord in vitro preparation.

Hemisected spinal cords from 10-14 day old anaesthetised rats were perfused with oxygenated modified $\mathbf{M g}^{++}$free Krebs. Spontaneous synaptic potentials were recorded from ventral roots pulled into suction electrodes. Remacemide and ARL12495 were superfused in $100 \mu \mathrm{M}$ strength for 20 minutes. For comparison, the NMDA antagonist D-aminophosphonovalerate (D-AP5, $50 \mu \mathrm{M}$ ) was also used. Data were captured as rate histograms and analysed by students $t$ test. The table summarises the results, which are shown as mean (SEM) frequency values; ${ }^{\star}$ denotes $P$ $<0.05$.

Endogenous $\mathrm{Mg}^{++}$blocks the NMDA receptor ion channel complex whereas its removal leads to unmasking of these channels. This is exhibited as more frequent and augmented synaptic activity. The data suggest that remacemide and ARL12495 significantly reduce this NMDA mediated activity. It is likely that remacemide may play an important therapeutic part in various 


\begin{tabular}{lll}
\hline & \multicolumn{2}{l}{ Spontaneous synaptic potentials $(\mathrm{Hz})$} \\
\cline { 2 - 3 } & $M g++$ free Krebs & $\begin{array}{l}\text { Mg++ free Krebs } \\
\text { with drugs }\end{array}$ \\
\hline Remacemide $(\mathrm{n}=7)$ & $3 \cdot 15(0 \cdot 07)$ & $2 \cdot 14(0 \cdot 06)^{\star}$ \\
ARL12495 $(\mathrm{n}=7)$ & $4 \cdot 93(0 \cdot 1)$ & $1 \cdot 65(0 \cdot 05)^{\star}$ \\
D-AP5 $(\mathrm{n}=3)$ & $2 \cdot 37(0 \cdot 05)$ & $0 \cdot 80(0 \cdot 03)^{\star}$ \\
\hline
\end{tabular}

neurological conditions in which NMDA receptors are implicated-for example, chronic pain syndromes.

MULTIFOCAL CEREBRAL T CELL

LYMPHOCYTOSIS PRESENTING WITH OPTIC NEURITIS

N Horwood, M Chowdhury, R Shakir, Charing Cross Hospital, London, UK

Cerebral lymphocytic infiltration can be seen in the context of demyelination granulomatous, and infective conditions, and cerebral lymphoma. A case is described which was not associated with any of these conditions, which presented with an acute optic neuritis.

A 41 year old man developed acute pain and blurred vision in the left eye, evolving over 48 hours, which resolved over four weeks. Visual evoked responses demonstrated left sided delay, with normal amplitude. Six months later he developed a progressive cerebellar and upper moto neuron syndrome. MRI showed multiple areas of high signal on $\mathrm{T} 2$ weighted images, in a predominantly subcortical distribution, including involvement of basal ganglia and cerebellum. CSF examination was normal. Three months later he developed rapidly progressive cognitive impairment with further deterioration in motor function Repeat MRI showed much more extensive signal change, including frontal and parietal cortices. Stereotactic brain biopsy revealed widespread perivascular lymphocytic infiltration, of polyclonal origin. There was no evidence of lymphomatosis, and no demyelinated plaques or gliosis. $\mathrm{He}$ responded rapidly to dexamethasone, and follow up MRI two months later shows partial resolution of the high signal lesions.

It is concluded that this patient has a steroid responsive inflammatory condition characterised by lymphocytic infiltration. The histopathology is not typical of demyelination, and the polyclonality of the cells argues against a lymphomatous condition.

VALUE OF THE SYMPATHETIC SKIN RESPONSE IN DETECTING AUTONOMIC DYSFUNCTION IN MULTIPLE SYSTEM ATROPHY

F Magnifico, VP Misra, NMF Murray, CJ Mathias, The National Hospital for Neurology and Neurosurgery, St Mary's Hospital/Imperial College School of Medicine, London, UK

The sympathetic skin response (SSR) measures a change in skin resistance to stimulation and thus determines sympathetic cholinergic activation of sweat glands. It has not been studied in adequate numbers of patients with MSA with documented autonomic failure (AF). We therefore performed the SSR in $32 \mathrm{MSA}$ along with 13 pure autonomic failure (PAF), and two siblings with dopamine- $\beta$-hydroxylase $(\mathrm{DBH})$ deficiency. 12 normal subjects were studied as controls. The diagnosis was confirmed on detailed autonomic testing. There were additional neurological features

(parkinsonian or cerebellar) in MSA. All patients had postural hypotension ( $>20 \mathrm{~mm}$ $\mathrm{Hg}$ systolic) and definite sympathetic failure. Sympathetic cholinergic function was preserved in DBH deficiency. None received drugs affecting the cholinergic system. SSR recordings were from palms and soles after auditory, tactile, and electrical stimuli and an inspiratory gasp.

SSR was present in all controls and in both patients with $\mathrm{DBH}$ deficiency. SSR was absent in all patients with PAF, and in 22 patients (of 32) with MSA. In each of the 10 patients with MSA with preserved SSR there was definite autonomic dysfunction; six had a disease duration of over four years.

In conclusion, the SSR was absent in PAF, and detected sympathetic cholinergic activity in DBH deficiency and controls. I was present in 10 patients with MSA. This could indicate an insensitive technique, or reflect the progressive nature of MSA and that sympathetic cholinergic function to sweat glands was spared despite evidence of sympathetic adrenergic failure. It thus has doubtful value, in isolation, in detecting autonomic dysfunction in MSA.

CLINICAL ASSESSMENT OF MUSCLE FORCE

A Goonetilleke, N Nikhar, SV Tan, RW Orrell, RJ Guiloff, Charing Cross Hospital, London, UK

Assessment of muscle force is important. Medical Research Council (MRC) scales are incompletely validated. Guidelines are provided for their use.

Dynamometry was performed on 16 muscle groups in 75 normals and 43 patients with motor neuron disease. Patients were also assessed using $6(0-5)$ and $8(4-$, 4, 4+) graded MRC scales by five neurologists. 4042 combined dynamometry and MRC grade assessments were analysed. Patients' dynamometry readings were expressed as \% normal force. For each MRC grade the corresponding mean \% normal force was defined as the MRCestimate.

Grades $0-3$ represented 10\% normal force. Upper, lower, and global limb scores based on MRC grades overestimated strength by $20-30 \%$. Intra and inter-rater reliability of 6 and 8 graded scales was good, with $\kappa$ coefficients of 0.63 and 0.66 respectively. MRC-estimates were more accurate, less variable and had a linear relation to dynamometry. Dynamometry slopes were similar to those with MRC grades and MRC estimates; change from baseline slopes were similar only to MRC estimates. Sample sizes were smaller using MRC estimates than

$\star P<0.001$.
MRC grades, the smallest being for change from baseline global scores. Sample sizes were increased by multiple raters; each rater should assess the same patients throughout.

Force scores and change from baseline slopes using MRC grades are inaccurate and require large sample sizes; conversion to MRC estimates improves these. Optimal longitudinal assessments are obtained with a single rater, using dynamometry or MRC estimates.

STURGE-WEBER SYNDROME, TRIGGERED BY PREGNANCY, PRESENTING IN THE FOURTH DECADE

JRC Bowen, ST Pendlebury, PTG Davies, Radcliffe Infirmary, Oxford, UK

Sturge-Weber syndrome (SWS) is regarded as a disease of childhood. A 33 year old woman with a facial angioma affecting the left forehead and upper eyelid presented in the 22nd week of her first pregnancy with convulsions, homonymous hemianopia, and dysphasia. Eclampsia was diagnosed and caesarean section performed. The convulsions, dysphasia, and hemianopia rapidly resolved after delivery although spontaneous visual phenomena (SVP) persisted in the previously hemianopic field. She represented weeks later with complex partial seizures, dysphasia, hemianopia, and headache. Ophthalmological assessment disclosed a left sided episcleral vascular anomaly with raised intraocular pressure. Computed tomography demonstrated one fleck of calcification in the left occipital lobe. Unenhanced MRI was unremarkable. Subsequent gadolinium administration disclosed florid cortical enhancement of the left temporoparietooccipital region and SPECT showed corresponding regional hypoperfusion characteristic of SWS.

Her complex partial seizures persist. Her dysphasia, hemianopia, and SVPs continue to fluctuate. The oldest age of presentation of SWS previously reported was 23 years. Little is known of the relation between pregnancy and SWS. Gadolinium enhanced MRI is required for thorough evaluation of cortical angiomas. SWS may present in the fourth decade with neurological symptoms in pregnant women with facial angiomas.

RESPONSIVENESS OF DISABILITY MEASURES IN MULTIPLE SCLEROSIS

JC Hobart, DL Lamping, JA Freeman, AJ Thompson, Institute of Neurology, London, UK

To enable sound disability measurement, instruments used must have proved reliability and validity. Ability to detect clinical change (responsiveness) is equally important if the instrument is to be used in therapeutic trials. This information is currently unavailable for most instruments. This study determined and compared the responsiveness of a range of disability measures, only one of which was disease specific (Kurtzke expanded disability status

\begin{tabular}{lll}
\hline Instrument & Effect size & $t$ value \\
\hline Patient report visual analogue scale & $0 \cdot 47$ & $3 \cdot 73$ \\
Barthel index & $0 \cdot 39$ & $7 \cdot 26{ }^{\star}$ \\
Functional independence measure & $0 \cdot 32$ & $7 \cdot 79^{\star}$ \\
Short Form-36 health survey (physical functions dimension) & $0 \cdot 25$ & $1 \cdot 69$ \\
Office of Population Census and Surveys disability severity & $0 \cdot 14$ & $1 \cdot 32$ \\
EDSS & $0 \cdot 06$ & $0 \cdot 96$ \\
Nottingham extended activities of daily living & $0 \cdot 06$ & $0 \cdot 83$ \\
\hline
\end{tabular}


scale (-EDSS)).

Methods-64 MS patients with moderate and severe disability admitted for inpatient rehabilitation were rated on admission and discharge with seven disability measures. Responsiveness was calculated in terms of an effect size and paired $t$ tests (increasing value $=$ increasing responsiveness).

Results-Mean age 42 range $21-69 ; 64 \%$ female; $80 \%$ secondary progressive disease; mean EDSS $7 \cdot 1$ range $5 \cdot 0$ to $9 \cdot 0$. The table shows the effect size and $t$ values.

Conclusions-Disability instruments vary in their responsiveness. The MS specific EDSS is considerably less responsive than some generic disability measures.

COMPARATIVE EVOLUTION OF ALZHEIMER'S DISEASE AND VASCULAR AND MIXED DEMENTIA

JV Bowler, M Eliasziw, R Steenhuis, DG Munoz, R Fry, H Merskey, VC Hachinski, University of Western Ontario, London, Canada and Charing Cross Hospital, London, UK

The neuropsychological changes in Alzheimer's disease (AD), vascular dementia $(\mathrm{VaD})$, and mixed dementia (MXD) have rarely been compared directly, although these profiles may help in the differential diagnosis. The evolution of $\mathrm{AD}, \mathrm{VaD}$, and MXD dementia were compared by cognitive domain using the University of Western Ontario dementia study.

129 patients with $\mathrm{AD}, 12$ patients with $\mathrm{VaD}$ and 36 patients with MXD were grouped to early, moderate, and advanced disease using the extended scale for dementia (ESD). This was subdivided into cognitive domains and the domain scores compared for each stage of disease by diagnosis using a two way analysis of variance with repeated measures.

The decline in memory differed between diagnostic groups $(P=0.024)$, mostly due to the difference between $\mathrm{AD}$ and $\mathrm{MXD}$ ( $\mathrm{P}$ $=0.026$ ), the difference between $A D$ and VaD only approaching significance $(P=$ $0.061)$. There was a similar finding for praxis. The interaction between diagnosis ( $\mathrm{AD}$ and $\mathrm{VaD}$ ) and severity was significant for memory $(P=0.024)$, showing a less severe memory deficit at onset but a proportionately more rapid progression in $\mathrm{VaD}$, and for arithmetical ability (AD and MXD ( $P=0.029)$ ).

Only memory impairment evolves differently between $\mathrm{AD}$ and $\mathrm{VaD}$, this depending on the severity. Memory is more severely impaired in early $A D$, but with increasing dementia severity, memory impairment in $\mathrm{VaD}$ accelerates and catches up with $\mathrm{AD}$ at the level of moderate impairment. Simple neuropsychological tools such as the ESD may be incapable of distinguishing between $\mathrm{AD}$ and $\mathrm{VaD}$.

ACUTE HEMIPLEGIC ISCHAEMIC STROKE CAUSES CONTRALATERAL QUADRICEPS WEAKNESS

ML Harris, MI Polkey, PMW Bath, J Moxham, King's College Hospital, London, UK

Muscle weakness in the unaffected limb after acute stroke could impair effective rehabilitation. Immobility and feeding difficulties are common after stroke and are known to cause muscle weakness. Using the technique of magnetic stimulation of the femoral nerve the strength of the quadriceps muscle was measured on the unaffected side in 10 patients with acute stroke both on admission and seven days later. 10 healthy elderly volunteers matched for sex and weight served as controls. The median (95\% confidence interval) change over one week, expressed as a percentage of baseline, was $-15 \%(-7$ to $-32 \%)$ in the stroke group and $+2.4 \% \quad(+0.7$ to $+4 \cdot 2 \%)$ in the controls. The difference between the groups was significant $(P<0.01$; Mann-Whitney $U$ test). There was a significant correlation between the percentage fall in quadriceps strength and both change in motor score ( $r s$ $=0.83, P<0.01)$ and percentage change in body weight $(r s=0.83, P<0.01)$. It is concluded that after acute hemiplegic stroke there is strength loss in the contralateral quadriceps; this may have important implications for effective rehabilitation.

\section{ISOLATED MYOCLONUS OF PLATYSMA}

MA Macleod, A Goonetilleke, C Fisher, PK Newman, Middlesbrough General Hospital, Middlesbrough, UK

Focal and segmental myoclonus can present as a manifestation of localised lesions within the neuraxis, from the peripheral nerve to the motor cortex. Palatal myoclonus, first described over 150 years ago, is perhaps the best known focal myoclonic entity. Isolated myoclonus of platysma, however, has rarely been described.

A 56 year old black African man, with a five year history of increasing stiffness and restriction of neck movements, presented with nine months of intermittent twitching in the neck muscles. On examination he had a reduced range of cervical spine movements and asynchronous, bilateral myoclonic activity of platysma (see video). Neurological examination was otherwise unremarkable. MRI showed cervical spondylosis with root compression at C3-7. Simultaneous EMG recordings showed asynchronous bursts of involuntary muscle activity isolated to platysma, more pronounced on the right.

Putative mechanisms of aetiopathogenesis and treatment options are discussed.

REGIONAL CEREBRAL BLOOD FLOW STUDIES IN PATIENTS WITH MIGRAINE: AN INTERICTAL STUDY USING SPECT AND TC 99M HMPOA F Ahmed, K Nijran, BE Jones, TJ Steiner, Charing Cross and Westminster Medical School, London, UK

There is evidence that $\mathrm{rCBF}$ is altered during migraine attacks, with interictal vascular dysregulation in response to physiological stimuli, more so in migraine with aura. Interictal studies of rCBF are few and inconclusive, most relying on visual analysis. As changes may be subtle, there is need for more sensitive methods.

70 patients with migraine satisfying International Headache Society diagnostic criteria were studied, $30(5 \mathrm{M}, 25 \mathrm{~F}$; mean age $38.9(13.4)$ years) without aura (MO) and $40(8 \mathrm{M}, 32 \mathrm{~F}$; mean age 42.5 (14) years) with aura (MA). The control group were 30 patients $(20 \mathrm{~F}, 10 \mathrm{M}$; mean age 35 (10) years) with episodic tension type headache.

A semiquantitative analysis of right-left asymmetry in regions of interest (ROI) were compared, an asymmetry index $>2.5 \mathrm{SD}$ from controls being abnormal. A group comparison was made using statistical parametric mapping (SPM).

Analysis with ROI showed asymmetries in up to $50 \%$ of patients with MA, mostly in posterior cerebral artery (PCA) territory. SPM showed areas of significantly $(P<$ 0.01 ) increased perfusion in occipital cortex and reduced perfusion in precentral gyrus. Patients with MO had few (< 10\%) asymmetries, with SPM showing no areas of altered perfusion.

Interictal abnormalities of rCBF are, therefore, more pronounced in patients with MA. Most are in PCA territory (and most patients with MA have visual aura). Patients with MO show few or no interictal abnormalities.

OLFACTORY EVOKED RESPONSES AND IDENTIFICATION TESTS IN NEUROLOGICAL DISEASE

CH Hawkes, BC Shephard, Ipswich Hospital, Ipswich, UK

To assess the value of smell testing olfactory evoked potentials (OEPs) were used with an identification test in multiple sclerosis; Parkinson's disease; motor neuron disease; and Alzheimer's disease.

Methods-The OEP to $\mathrm{H}_{2} \mathrm{~S}$ (20 ppm) was obtained with an olfactometer designed to stimulate olfactory nerve endings only. Odour recognition was assessed by the University of Pennsylvania smell identification test (UPSIT). In all instances the disease was "definite" based on standard diagnostic criteria. Controls were derived from 154 healthy members of hospital staff or British Telecom. A result was judged abnormal if it was outside $95 \%$ limits for our control population.

Results-(1) Multiple sclerosis-11/72 patients $(15 \%)$ were abnormal on UPSIT. For OEP there was significant increase of latency and decrease in amplitude in 6/26 patients $(23 \%)$. (2) Parkinson's disease$126 / 155(81 \%)$ patients had abnormal UPSIT score. $12 / 37(32 \%)$ had prolonged latency with normal amplitude measurement on OEP but 27 had absent or unclear readings. 4/10 with normal UPSIT displayed abnormality on OEP. (3) Motor neuron disease-9/58 (16\%) were abnormal on UPSIT. There was significant delay in $1 / 10(10 \%)$ patients on OEP. (4) Alzheimer's disease-UPSIT scores were abnormal in all eight patients examined. OEP was normal in four of these who could be tested.

Conclusion-Smell dysfunction was found in all four conditions but most severely in Parkinson's disease (over $80 \%$ ). The UPSIT in general showed abnormality more often than OEP. The olfactory defect probably involves peripheral structures in all diseases tested except Alzheimer's. A patient with normal olfaction is unlikely to have idiopathic Parkinson's disease.

DIPOLE SOURCE LOCALISATION: A

COMPARISON OF BRAIN ELECTRICAL SOURCE ACTIVITY AND ELECTROMAGNETIC SOURCE ESTIMATION

CEG Moore, W Schady, Manchester Royal Infirmary, Manchester, UK

The accuracy of two methods of dipole source localisation, BESA (brain electrical source activity) and EMSE (electromagnetic source estimation) have been compared. In computing a dipole source BESA constrains the number of data points and thus forces the low pass filter to be maximally set at $\mathbf{5 0 0}$ Hz. This study assessed the effect of this 
restrictive filtering on the waveforms of the Somatosensory evoked potentials (SEPs) and on the size and position of the resulting dipole source using EMSE. SEPs were recorded in five subjects. $1000200 \mathrm{~ms}$ pulses, were delivered to the median nerve at the wrist $(1 \cdot 8 \mathrm{~Hz})$. Responses were recorded from 30 scalp electrodes referenced to the ipsilateral ear lobe, band pass filtered $(1-2000 \mathrm{~Hz})$, and stored digitally. Off line manipulation of the data occurred in four ways: A-filter (F) 1-2000 $\mathrm{Hz}$, reference (R) mastoid; $B-(F) 1-2000$ $\mathrm{Hz}$, (R) Common average; C-(F) 25-500 $\mathrm{Hz}$, (R) mastoid; D-(F) $25-500 \mathrm{~Hz}$, (R) average. These parameters were the most commonly used in previous reports on SEP source localisation. In comparison with $\mathrm{A}$ $\mathrm{B}, \mathrm{C}$ and $\mathrm{D}$ all resulted in a decrease in the peak to peak amplitude of the N20-P27 waveform by $4 \cdot 2-33 \cdot 7 \%$ (mean $15 \cdot 2 \%$ ). The effect of filtering being greater than the effect of changing reference method. The position of a single dipole modelled to the peak of the $\mathrm{N} 20$ wave varied up to $3 \mathrm{~cm}$ away from the dipole calculated in $\mathrm{A}$ and the dipole moment changed by up to $50 \%$. Whereas BESA remains the most published source analysis tool, the accuracy of the results must be carefully scrutinised, especially if the evoked response contains significant energy above $500 \mathrm{~Hz}$ as in the SEP.

TECHNIQUES FOR THE NEUROPHYSIOLOGICAL EVALUATION OF RESPIRATORY FUNCTION

VP Misra, RS Howard, BD Youl, The Royal London Hospital and the National Hospital for Neurology and Neurosurgery, London, UK

A number of techniques are available for the neurophysiological investigation of patients with respiratory dysfunction as a result of neuromuscular disease. The evaluation of many of these approaches is incomplete; those which are regularly employed in the above institutions are detailed.
Nerve conduction techniques

(1) Phrenic nerve conduction studies, which are generally well tolerated, yield reproducible distal latency and compound muscle action potential values. (2) Intercostal nerve stimulation employs an indwelling active recording electrode and stimulation at two proximal sites.

Needle EMG techniques

These have focused on the diaphragm and intercostal muscles. Denervating and myopathic conditions are usually readily distinguishable. Patients with myotonic dystrophy are of particular interest, in that they may exhibit both profuse myotonic discharges and myopathic features. Single fibre examination of the diaphragm is not feasible on technical grounds; however, the intercostal muscles have proved amenable to study in this way. This procedure may prove a useful adjunct to the investigation of patients with myaesthenia gravis.

PERSISTENT HEADACHE AFTER DIAGNOSTIC LUMBAR PUNCTURE

KAC Harkness, HL Ford, St James's

University Hospital, Leeds, UK

Postdural puncture headache is a well recognised complication of both spinal and epidural anaesthesia. Persistent headache, defined as headache of greater than one year's duration, has been reported in up to $3.7 \%$ of patients after spinal anaesthesia and in $15 \%$ of obstetric patients who have had accidental dural puncture during epidural anaesthesia. The use of larger gauge needles for epidural (16G) compared with spinal anaesthesia (24/26G) has been thought to be contributory. There have been no studies investigating the incidence of long term complications after diagnostic lumbar puncture (LP).

A pilot postal questionnaire study was carried out on patients with multiple sclerosis who had had diagnostic lumbar puncture, to assess the occurrence of persistent headache. The temporal relation to LP was derived from the case records. The questionnaire was designed to differentiate between tension headache, migraine, and low pressure headache. 94 patients from a prevalent population registrar of multiple sclerosis were surveyed The length of time from LP was from 12 months to 10 years. $68 \%(64 / 94)$ of patients responded of whom $32(50 \%)$ had no headaches, $24(38 \%)$ described tension headaches, and seven (11\%) had features of migraine. One patient had an 18 month history of low pressure headache with onset within one week of diagnostic LP. The procedure had been atraumatic and uncomplicated.

The pilot study suggests that persistent headache may be a cause of unrecognised morbidity after diagnostic LP.

THE CHANGING FACE OF REFLEX EPILEPSY DF Scott, London, UK

Alteration in the pattern of reflex, sensory evoked, stimulus sensitive, epilepsy is reported as a result of a literature study and a survey of clinicians' experience. Though definite accounts of this disorder appear in the late 19th century, it was the 1940s when the use of the EEG enabled detailed investigations to be carried out. The occurrence of photosensitive epilepsy has, and remains, of major importance, as well as television induced seizures, pattern, and colour factors are now recognised.

By the 1950s and 1960s, eating, musicogenic, and reading seizures had been reported. More recently, hot water induced types have been noted and precipitation by calculation and games. Particularly in the 1980s, video induced seizures became important. Clinicians currently vary in their reports on the occurrence of reflex forms of epilepsy, but a decrease in incidence is common, with the exception of photosensitive types. No definite reasons emerge. However, the introduction of newer antiepileptic drugs seems to be a likely major factor. 